\title{
Wear issues of pumping units
}

\author{
Oybek Ishnazarov ${ }^{1 *}$, Abdusaid Isakov $^{2}$, Utkir Islomov $^{2}$, Umirzoq Xoliyorov $^{2}$ and Dilshod \\ Ochilov $^{3}$ \\ ${ }^{1}$ Scientific and Technical Centre of JSC Uzbekenergo, Tashkent, Uzbekistan \\ ${ }^{2}$ Tashkent Institute of Irrigation and Agricultural Mechanization Engineers, Tashkent, Uzbekistan \\ ${ }^{3}$ Karshi branch of the Tashkent Institute of Irrigation and Agricultural Mechanization Engineers, \\ Uzbekistan
}

\begin{abstract}
The article presents the possibility of increasing the service life of pumping units. Particular attention is paid to the regulation of the speed of rotation of the pumping unit. There are some assumptions in the mathematical model that do not affect the final result. The factors influencing the operating mode are given. It is indicated that the speed of rotation of the pump shaft significantly affects the wear resistance of the pump blades. Thus, the regulation of the pump rotation speed will rationally increase its service life.
\end{abstract}

\section{Introduction}

The efficiency of the technological lines of mining and metallurgical enterprises is largely determined by the stability of the operating modes of centrifugal pumps.

However, during operation, insufficient durability of the pumps is noted, which is caused by increased hydroabrasive wear. This disadvantage is especially evident when processing highly abrasive soils.

To assess the effective operation of a centrifugal pump, an indicator is required that considers the technical condition of the mechanism and its ability to meet technological requirements and the economic feasibility of operating it in a controlled mode. This indicator can be the amount of wear of pump parts. During operation, pump parts (blades, armored discs, etc.) are in contact with soil particles, which lead to intense hydroabrasive wear [1-4].

\section{Materials and Methods}

Hydroabrasive wear is understood as the destruction of parts of the flow path of hydraulic machines due to the mechanical action of solid particles of the working fluid. In the process of destruction, the shape and linear dimensions of the parts change. Therefore, concerning turbo mechanisms, the total value of destruction during abrasive wear changes by a decrease in the pump's volume and weight [1-5].

Destruction occurs due to continuous collisions, transported by the flow of solid particles with the part's surface. At the moment of collision, the kinetic energy of the

*Corresponding author: oybek.ishnazarov@gmail.com 
moving particle is converted into the work of deformation of the material of the streamlined part. In case of residual deformations, the particles of the surface layer will be separated from the bulk of the part, leaving a trace that has significant roughness due to the nature of the impact, crystal structure, and inhomogeneity of the metal. The countless collisions of solid particles transported by the flow with the surface of the part, even if they only cause elastic deformations of the material, also ultimately lead to surface destruction due to the phenomena of metal fatigue [6-9].

Due to the wear of the impeller of the dredge pump, the size of its blades decreases, and the impeller stops developing the design head and productivity. Thus, there is a change in the pressure characteristics, a decrease in the efficiency of the pump; because the gaps and losses increase, the effective cross-section of the working cavities decreases, which leads to an increase in electricity consumption for pumping liquid. As a result, a moment comes when the turbomachine's parameters cease to meet the technological requirements.

According to research results [10-12], if pump parts have lost $20-25 \%$ of their original weight due to wear, their replacement or restoration is required.

According to [13-14], on average, for every $1000 \mathrm{~m} 3$ of soil processed by hydromechanization, about $0.75 \mathrm{~kg}$ of metal of pump parts is lost due to wear. The cost of wear is the cost of the parts and the cost of downtime for the dredger. For example, as experience has shown, the limiting wear of the $8 \mathrm{Gr}-8$ dredge pump causes a decrease in pressure by $10-15 \%$ and productivity by $30 \%$ or more.

As the pump wears out, its operating characteristic changes. This is mainly due to changes in the hydraulic and volumetric losses in the pump, which result from changes in the size of the main organs of the dredge pump during wear. The development of imbalance of the impeller from uneven wear of its blades causes an increase in power consumption. The main part of consumable spare parts is impellers (45-50\%), housings $(25 \%)$, and armored discs (less than 25\%).

As a result of the analysis of sources, it was found that the wear rate of dredge pumps is not the same in different periods of its operation, which can be divided into three periods:

- the running-in period of parts, characterized by intense wear, a short period;

- the period related to the normal operation of the unit, and wear occurs relatively slowly during this period;

- the period of the most intensive wear and wear is relatively fast.

In this regard, it can be concluded that the wear rate at the beginning of operation is relatively low; then, due to the weakening of the stressed state of the surface layer and the appearance of microcracks, it increases significantly. This allows us to assume that the rate of wear of the mineral-polymer coating depends on the degree of damage.

\section{Results and Discussion}

Numerous scientific research works indicate the possibility of a significant increase in the wear resistance of turbine mechanisms by making their working bodies from special materials and improving the designs and shapes of the pump channel capable of withstanding the effects of abrasive wear.

Table 1. It is the data on the mean time between failures (h) of parts and assemblies of the $8 \mathrm{Gr}-8$ pump

\begin{tabular}{|l|c|}
\hline \multicolumn{1}{|c|}{ Details } & operating time (h) \\
\hline Working wheel & 550 \\
\hline Diversion & 700 \\
\hline Front bearing & 680 \\
\hline Rear bearing & 910 \\
\hline
\end{tabular}


The wear of pump parts is complicated by the presence of many additional factors, for determining which it is currently impossible to select exact mathematical relationships. Continuous change and pulsation of velocities and pressures during the flow of the slurry through the elements of the flow path of the turbine mechanism, the separation of the flow into several separate streams, the uneven separation of the velocities along the sections, the presence of sharp turns, the inhomogeneity of the composition of suspended particles, variable operating modes-all this extremely complicates the actual picture of hydroabrasive wear.

The wear rate of the pump unit is influenced by several factors, namely: size, hardness, and relative speed of soil particles; the angle of the meeting of soil particles with a wearing part; pulp flow density; wear resistance of the part material, i.e.

$$
E=f(v, \rho, \delta, \lambda, \mu, \beta, \alpha)
$$

where $v$ is the pulp flow rate, $\mathrm{m} / \mathrm{s} ; \rho$ - pulp density, $\mathrm{kg} / \mathrm{m}^{3} ; \delta$ is ultimate strength of the wearing part; $\lambda$ is hardness of the wearing part, $P a ; \mu$-hardness of particles in the pulp, $\mathrm{Pa} ; \beta$ is design of pump parts in contact with the slurry; $\alpha$ is the angle of attack of the pulp flow velocity vector.

The operation of pumping units in the hydrometallurgical industry shows that the amount of wear depends on the following factors: with an increase in the particle diameter, the wear rate first increases rapidly, then grows slowly in the size range from 1-1.5 to 20-25 $\mathrm{mm}$, and after the particle size particles exceed $25 \mathrm{~mm}$, a rapid increase in intensity begins again; with the roundness of the particles, the wear rate first drops rapidly and then slowly; with an increase in the hardness of the particles, the wear rate at first grows slowly, then when the hardness of the soil particles becomes equal to the hardness of the material of the part, the wear rate grows very quickly, but after the hardness of the soil particles becomes significantly greater than the hardness of the part, the wear rate stops increasing; the wear rate is inversely proportional to the wear resistance of the part material; the wear rate increases very quickly with an increase in the particle speed and the increase in wear rate is proportional to the third power of the speed; with an increase in the pulp density, the wear rate initially increases very quickly, but from a certain moment, depending on the size of the pumped material, the density ceases to be reflected in the wear rate; the intensity of wear, depending on the angle of meeting of the pulp flow with the surface of the part, first increases to a certain angle and then begins to fall.

Of all the listed factors (1) affecting hydro abrasive wear, significant, from the point of view of the impact on the wear process by choosing a rational mode of operation of the pumps, is - the density of the pumped slurry $\left(\mathrm{kg} / \mathrm{m}^{3}\right)$ and - the slurry flow rate, the slurry flow rate, $\mathrm{m} / \mathrm{s}$.

The density of the pulp flow is determined by the requirement of the technological process and is $1400 \mathrm{~kg} / \mathrm{m}^{3}$. The experience of slurry pumping pumps shows that in the practice of operating the slurry pumping system, changes in the density of the pumped slurry are possible. As a result, the system starts to operate in a transient mode with changed parameters. Calculations show, as shown in Figure 1 that with an increase in the density of the pulp, the power consumption can exceed the permissible for a very long time, and with a decrease in the density of the pulp, the speed of the pulp in the pipeline may decrease with the danger of blocking the pipeline. Also, increased pulp density is accompanied by increased wear, but the proportion of crushed ore pumped out increases compared to low density pulp pumping as a function of time. 


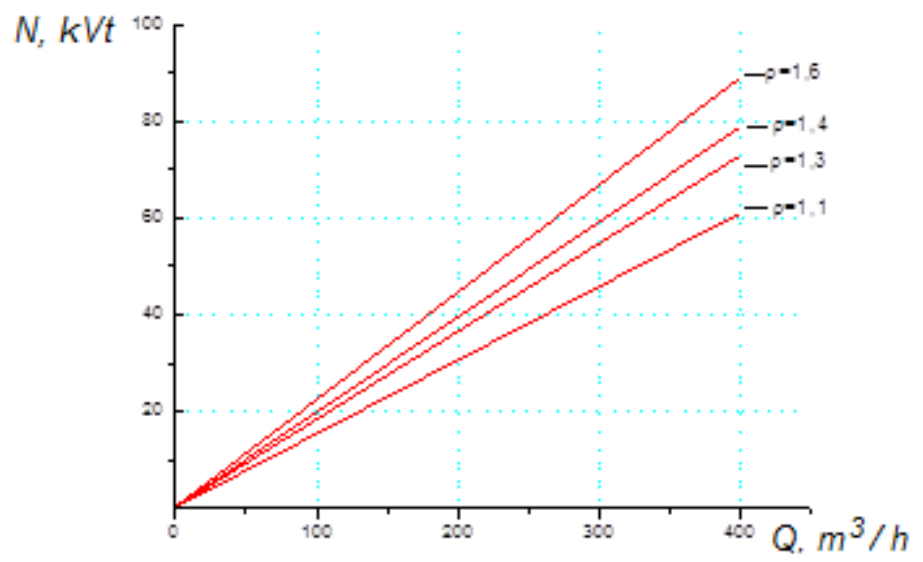

Fig. 1. Influence of pulp density on pump power consumption: -power consumption; - productivity; pulp density $\mathrm{t} / \mathrm{m}^{3}$.

In variable mass hydraulics, it is proved that the equations of dynamics of variable mass can be replaced by a single equation

$$
\frac{d(m u)}{d t}=F-\frac{d m_{1}}{d t} u_{1}+\frac{d m_{2}}{d t} u_{2}
$$

where $m$ is the total mass; $\mathrm{u}$ is the average flow rate; $t$ is time; $F$ is the projection of the resultant forces on the direction of the velocity of one jet; $u_{1}, u_{2}$ are projections of the velocities of the detached and attached masses.

In the case under consideration, the detachable mass is the pulp flowing out of the pipeline with a density $\rho_{1}$. The added mass is the pulp entering the system with a density $\rho_{2}$.

For a pipeline with a constant diameter $u_{1}=u_{2}=u$, the initial mass is

$$
m_{\text {nach }}=s L \rho_{1}
$$

where $s$ is the cross-sectional area of the pipeline; $L$ is the length of the pipeline. Equation (2) taking into account (3) we obtain

$$
\left(s L \rho_{1}-s l \rho_{1}+s l \rho_{2}\right) \frac{d u}{d t}=F_{n}-F_{t r}
$$

where $F_{H}$ is the force causing the slurry to move due to the pump head; $F_{t r}$ is force of resistance to the movement of the pipe in the pipeline; $l$ is the distance from the beginning of the pipeline to the moving boundary of the pulp density change. The forces $F_{t}$ and $F_{t r}$ are expressed through the pump head and the pressure loss in the pipeline. Then we get

$$
\left(s L \rho_{1}-s l \rho_{1}+s l \rho_{2}\right) \frac{d u}{d t}=H_{n}-H_{t r 1}-H_{t r 2}
$$


where $H_{n}$ is pump head as energy per unit volume of liquid; $H_{t r l}$ is pressure losses on the part of the pipeline length $(L-l)$, filled with pulp density $\rho_{1} ; H_{t r 2}$ is the same for the rest of the pipeline length with slurry density $\rho_{2}$.

The analysis of the operating mode with the changed pulp density shows that when the limit of the pulp density change reaches the pump, its head increases due to the increase in the pulp density in the pump. However, the pipeline at this time is still filled with slurry with a lower density, and the pressure loss in the pipeline has not yet significantly increased. As a result, there is a temporary excess of the pump head over the head loss in the pipeline and, as a result, a temporary increase in the slurry flow rate. In the future, as the border of the change in the density of the pulp moves along the pipeline, the pressure loss will increase, and the flow rate of the pulp will begin to decrease. In the transient mode, caused by a decrease in the density of the pumped pulp, a similar temporary decrease in the pulp consumption occurs against the background of its general increase. Such a temporary decrease in flow rate can lead to a decrease in the pulp velocity below critical.

Studies of the kinematics of movement of large abrasive particles in the pump have shown that their relative velocity at the moment of impact on the leading edges of the blades is close in magnitude to the peripheral velocity of the latter. Naturally, to increase the service life of the blades, it is necessary to reduce the peripheral speed of their input edges and, first of all, of the part that adjoins the rear disc of the wheel.

The peripheral speed of the leading edges of the blades can be reduced in two ways: by reducing the number of revolutions of the impeller and by displacement in the design of the considered elements on a circle of smaller diameters. The first of these methods relate to the operating mode of the pumps, and the second to the design of the impellers. We are considering the first method, in which the stabilization of operating modes of pumping units with dredge pumps is mainly associated with the possibility of regulating their operating modes.

From expression (1) it follows that wear is determined by several interrelated factors. But the most significant, from the point of view of the possibility of influencing the wear process, is the pulp flow rate $-v$. The wear process can be influenced by adjusting the pump shaft speed. It is known that the slurry flow rate is a function of the impeller speed, i.e. $v=f(n)$, and the pump wear rate is proportional to the cube of the pump speed $n$

$$
E=A n^{3}
$$

where A is the proportionality coefficient.

In connection with (6), the main direction in increasing the wear resistance of pumps is to reduce the speed of rotation of the pump shaft.

Our studies of the pumping equipment operation showed that the $8 \mathrm{Gr}-8$ dredge pumps used are subject to significant wear, and their service life without replacing the corundum coating is 400-600 hours. Figure 2 shows the dependence of the wear time on the speed of rotation of the pump shaft, i.e., for pump $8 \mathrm{Gr}-8$. Calculations show that when the pump operates at the minimum rotation speed, taking into account the slurry rise by, the pump service life increases more than 6 times. 


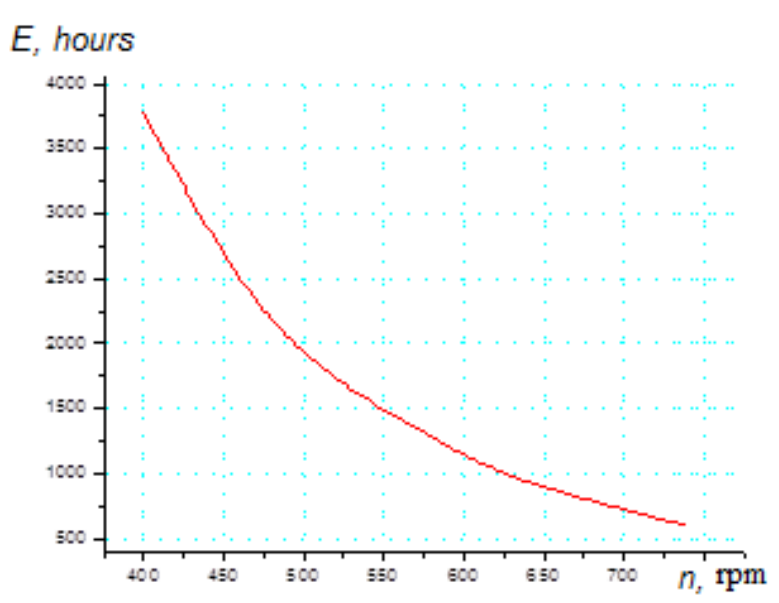

Fig. 2. Dependence of the wear time of the pump $8 \mathrm{Gr}-8$ on the speed of rotation of the pump shaft.

Taking into account the control range, we determine the minimum rotation speed $n_{\min }$ [15-16].

$$
\frac{H_{s t}}{H_{1}}=\left(\frac{n_{\min }}{n_{n o m}}\right)^{2}, \text { from where } n_{\min }=n_{n o m} \sqrt{\frac{H_{s t}}{H_{1}}}
$$

where $H_{s t}$ is the static head, $\mathrm{m} ; H_{1}$ is pressure developed by the pump at $n_{n o m}, \mathrm{~m} ; n_{\text {nom }}$ is rated rotation speed, rpm.

Abrasive wear of the dredge pump leads to a change in the hydrotransportation mode. As our calculations have shown, the decrease in pump performance due to wear by 25 and $45 \%$ corresponds to the operation of the pump with a decrease in the rotation speed, respectively, to 570 and $420 \mathrm{rpm}$. Pump characteristics for these modes are shown in Fig. 3. It is shown that the change in the parameters of productivity, head, and efficiency is due to the deterioration of the hydraulic properties of the dredge pump as it wears out. Considering that the efficiency of the dredge pump determines the breadth of its industrial use, let us analyze its operation in the field of changing modes.

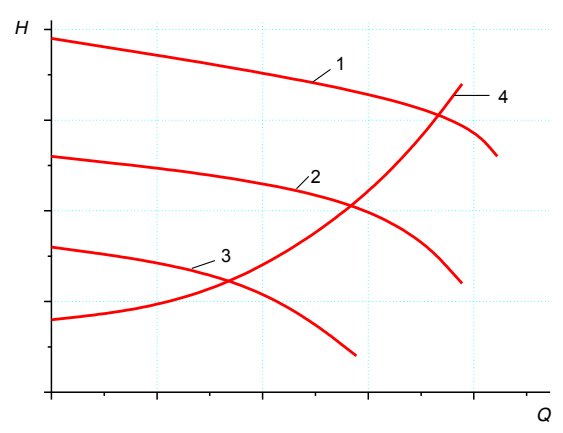

Fig. 3. The performance of the dredge pump during its wear: 1, 2, 3 are pressure characteristics, respectively, of a new one and with a decrease in productivity by 25 and $45 \%$ as a result of pump wear; 4 is characteristic of the pipeline. 
Let us denote the operating parameters of a dredge pump with a rotational speed through $H_{A}, Q_{A}$, and $\eta_{A}$. To estimate the efficiency of the dredge pump in modes $H_{C}$, $Q_{C}, \eta_{C}$, and, $n_{2}$ we will change the mode of its operation in two directions: at a constant value of the resistance of the external network with a change in the speed of rotation of the pump rotor from $n_{1}$ to $n_{2}$ (section of the $\mathrm{AB}$ curve, Figure 4.) and at a constant speed of rotation of the pump rotor with by changing the resistance of the external network (section of the $\mathrm{BC}$ ).

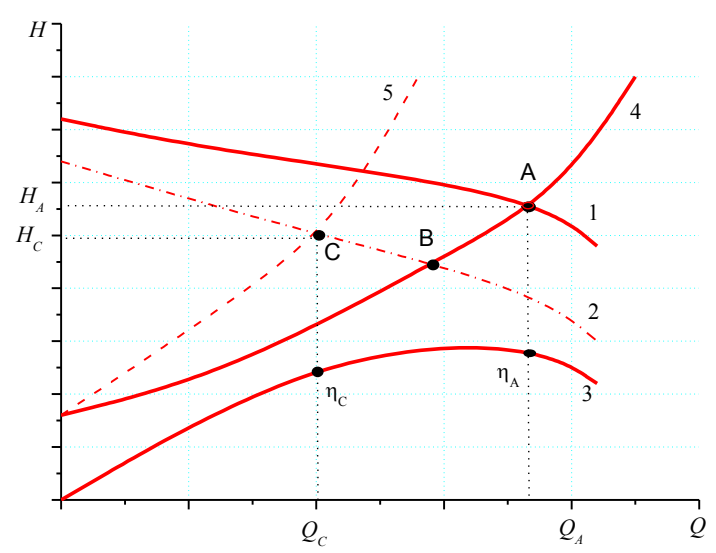

Fig. 4. Operating characteristics of the $8 \mathrm{Gr}-8$ dredge pump and pipeline: 1,2 are pressure characteristics of the pump with the rotor speed and; 3 is characteristic of the efficiency; 4,5 are characteristics of the pipeline with minimum and maximum resistance.

A decrease in the rotor speed of the dredge pump from $n_{1}$ to $n_{2}$ leads to insignificant changes inefficiency (from $\eta_{A}$ to $\eta_{B}$ ) due to the proportionality of losses in the seals and bearings, respectively, of the first and second degrees, while hydraulic losses are proportional to the third degree of the pump rotor speed.

The change in efficiency depending on the speed of rotation is determined using the Moody formula, transformed for pumps [17-18].

$$
\frac{1-\eta^{2}}{1-\eta_{1}}=\left(\frac{D_{1}^{2} n_{1}}{D_{2}^{2} n_{2}}\right)^{2}
$$

where $\eta_{2}, D_{2}$ are efficiency, rotation speed, and diameter of the pump impeller; $\eta, n_{1}$, $D_{1}$ are the same for a geometrically similar pump. After some transformations, we get

$$
\eta=1-\frac{\left(1-\eta_{\text {nom }}\right) n_{n o m}}{n}
$$


where $\eta_{\text {nom }}$ is the efficiency value at the rated pump speed $n_{n o m} ; n$ is current pump rotation speed. It should be noted that the pump efficiency at rated speed is not the rated efficiency. For the rated efficiency of the pump, only the maximum value of efficiency is taken at the rated speed of the pump impeller [17].

As you can see, a decrease in the speed of rotation of the dredge pump increases the wear resistance and the overhaul cycle of the pumps. But, one pump with a minimum rotation speed does not meet the requirements of the technological process. In this regard, to meet the technological process requirements, it is necessary to regulate the pump capacity at the dictating sump point. From an energetic point of view, the level of the pulp at the dictating point of the sump should be maintained at the highest point since this reduces the static lift height and, accordingly, the minimum rotation speed. This can be achieved through an appropriate control system.

Let us determine the economically justified limiting operating modes of dredge pumps. As noted, with the wear of the pumps, the power consumption and the energy consumption for pumping out the slurry increase, which leads to a decrease in the pump efficiency. It follows from this that the replacement of worn-out pump equipment is necessary when the cost of the excessive consumption of electrical energy from the operation of the pumping unit exceeds the cost of its repair. This problem can be solved by setting the loss of electricity when the pump is worn out:

$$
\Delta W_{n}=\frac{\rho Q H}{102 \eta} t\left(\frac{\eta_{\text {nom }}}{\eta_{i z m}}-1\right)
$$

where $\rho$ is the pulp density, $\mathrm{kg} / \mathrm{m}^{3} ; \eta_{\text {nom }}$ - nominal pump efficiency; $\eta_{i z m}$ - Efficiency of a worn out pump.

Figure 5 shows the dependence of electrical losses $(\mathrm{kW})$ for 600 hours of pump operation when it is worn out. If we assume that the cost of electricity losses $\Delta W_{1}=\Delta W_{n} A$, then replacement of worn-out equipment is necessary when $\Delta W_{1} \geq W_{11}+W_{12}$. Here $A$ is the cost of consumed electricity; $W_{11^{-}}$the cost of repair and replacement of pump elements; $W_{12}$ is the cost of newly installed equipment.

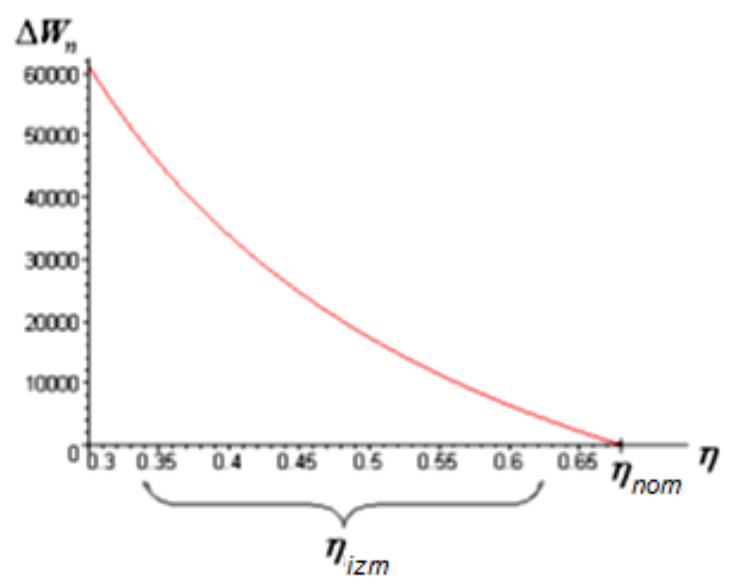

Fig. 5. Electrical losses of a worn out pump 


\section{Conclusions}

Thus, it has been determined that the amount of wear of pump parts is one of the main factors, taking into account the technical condition of the pump and its ability to meet technological requirements and the economic feasibility of its use.

It has been established that the operation of $8 \mathrm{Gr}-8$ pumps in the mode of the minimum rotation speed $n_{\min }$, taking into account the control range, will increase the service life of the pumps without replacing the corundum coating by more than 6 times.

\section{References}

1. Karelin V.Ya. Wear of vane hydraulic machines from cavitation and sediment, Mechanical engineering, 184, p. 126. (1970)

2. Shkundin B.M. Machines for hydromechanization of earthworks p.183, Moscow, Stroyizdat, (1982)

3. Ovchinnikov N.P. Experimental studies of the influence of hydroabrasive wear of various degrees of impeller elements on the vibration state of the electric pump unit. Mining information and analytical bulletin (scientific and technical journal), (1), 158165. (2018)

4. Isakov A., Rakhmatov A. IOP Conf. Ser.: Mater. Sci. Eng. 883 012118. (2020)

5. Isakov A., Abdullaev Z. IOP Conf. Ser.: Earth and Environmental Science 614 012047. (2020)

6. Ovchinnikov N.P. Problems of operation of sectional type electric pumping units at the ALROSA kimberlite mines and ways to solve them. Bulletin of the Tomsk Polytechnic University. Engineering of georesources, 329 (6), 66-73. (2018)

7. Tarasov E.V., Kostyukov V.N. Control of staged degradation of parts and assemblies of centrifugal pumping units in operation "Dynamics of systems, mechanisms and machines, 1 (1), pp. 115-121, (2016)

8. Koroli M, Ishnazarov O. Mathematical modeling of a heat pump and its operation modes, E3S Web of Conferences 216, 01165, (2020)

9. Isakov A., Rakhmatov A., Ismailova Z. IOP Conf. Ser.: Earth and Environmental Science 614 012011. (2020)

10. Romanenko V.A. Failure model in case of hydroabrasive wear of dredge pump casings. Gornyi Zhurnal, 1, pp. 42-43, (1986)

11. Isakov A., Mirzabaev A., Sitdikov O., Makhkamova M., Kodirov D. IOP Conf. Ser.: Earth and Environmental Science 614 012014. (2020)

12. Mirzabaev A., Isakov A., Mirzabekov S., Makhkamov T., Kodirov D. IOP Conf. Ser.: Earth and Environmental Science 614 012016, (2020)

13. Suprun V.N. Abrasive wear of dredge pumps and the fight against it, M. Mechanical Engineering, p. 104, (1972)

14. Muzafarov S., Balitskiy V., Toqaev B., Batirova L., Isakov A. IOP Conf. Ser.: Earth and Environmental Science, 614 012008. (2020)

15. Ishnazarov O. Kh. Simulation model of frequency-controlled electric drives for slurry pumps in the mining industry, Problems of Informatics and Energy, 6, pp.58-65, (2009)

16. Isakov A.Z., Bugakov A.G. Applied Solar Energy (English translation of Geliotekhnika) 50(3), pp. 188-190, (2014)

17. Leznov B.S. Saving energy in pumping units. M.: Energoatomizdat. p. 144 (1991)

18. Isakov A.Z. Applied Solar Energy (English translation of Geliotekhnika) 46(1), pp. 77-79, (2010) 